\title{
A Study of Shoulder Stabilizer Muscle Exercise using the Contraction of the Finger Flexor Muscle
}

\author{
Sangyeol Lee, PhD, $\mathrm{PT}^{1}$, Wontae Gong, PhD, PT ${ }^{2)}$, Minchull Park, PhD, $\mathrm{PT}^{3}$, \\ MyoungheE LeE, PhD, PT ${ }^{4)}$, JeMYUng ShIM, PhD, $\mathrm{PT}^{1)}$ \\ 1) Department of Physical Therapy, Gimhea College \\ ${ }^{2)}$ Department of Physical Therapy, Gumi College: 407, Bugok-dong, Gumi-si, Kyoungbuk, 730-711 \\ Republic of Korea.TEL: +82 54-440-1244,FAX: +82 54-440-1179,E-mail:owntae@hanmail.net \\ ${ }^{3)}$ Department of Physical Therapy, Pusan National University Hospital \\ 4) Department of Physical Therapy, Daegu Health College
}

\begin{abstract}
Purpose] The purpose of this study was to evaluate the effect of shoulder stabilizer muscle activity using the contraction of the finger flexor muscle. [Subjects] We divided the study subjects into a grasp group $(\mathrm{n}=17)$ and a non-grasp group $(n=17)$. [Methods] The shoulder muscle activities of both the grasp group and the non-grasp group were measured by electromyography. [Results] Both the grasp group that used the contraction of the finger flexor muscle and the non-grasp group that did not use the contraction of the finger flexor muscle showed a state where shoulder stabilizer muscle activation increased as the weight borne by them increased. The serratus anterior muscles and rhomboid muscles showed differences between the two groups. [Conclusion] Exercises using finger flexor muscle activation is a more effective method for patients who need shoulder stabilizer muscle strengthening exercises because of shoulder stabilizer muscle weakness.
\end{abstract}

Key words: Electromyography, Shoulder stabilizer, Stability

(This article was submitted May 25, 2010, and was accepted Jun. 22, 2010)

\section{INTRODUCTION}

To provide stability when the shoulder complex is moving, the upper and lower trapezius muscles and the serratus anterior muscles should work together as a double force. The rhomboid muscle also plays an important role in providing stability to the surrounding shoulder areas ${ }^{1)}$. Sahrmann reported that the characteristics of scapular movement impairment syndromes resulting from abnormal scapular and humeral motions cause pain in certain areas ${ }^{2)}$. Increases or decreases in the range of motion of joints and unbalanced weakness of the shoulder stabilizer muscle might also occur. Bleuse et al. reported that they also for stable movements of distal parts, anticipatory control of proximal parts should be included ${ }^{3)}$. He reported that this anticipatory control was to increased the stability of proximal parts in free movements of distal parts, and this was an essential element of distal part movements ${ }^{4}$. This means that exercises to stabilize the shoulder complex are important for movements of distal parts such as the wrists, elbows and fingers. Because these shoulder complex stabilizing exercises are known to be directly related to postoperative recovery, they are also very important in rehabilitation both before and after total shoulder arthroplasty $^{5}$.

Studies of diverse exercising methods have been conducted in order to find ways to improve shoulder stability by enhancing shoulder stabilizer muscle activation. In particular, push up plus exercises $\left.{ }^{6}\right)$ using closed kinetic chains and exercises in open kinetic chains using TheraBands are frequently used ${ }^{7}$. Many studies have been conducted on both open kinetic chains and closed kinetic chains. However, the current knowledge about proximal part muscle activation related to distal part muscle activation using the occurrence of anticipatory control in open kinetic chain exercises remains insufficient.

Consequently, this study is intended to examined the effect of activating the finger flexor muscle, as a way of enhancing the activity of the shoulder complex stabilizer muscle, in relation to the activity of the finger flexor muscle in shoulder abduction and adduction.

\section{SUBJECTS AND METHODS}

Study subjects were selected from normal adult males in their 20s with no musculoskeletal disease in the shoulder complex and upper limbs and who had the normal ROM. The age of the grasp group $(\mathrm{n}=17)$ that used the finger flexor muscle was $26.13 \pm 2.42$. The Body Mass Index (BMI) of the grasp group was $23.11 \pm 2.13$. The age of the non-grasp group $(\mathrm{n}=17)$ was $27.15 \pm 1.42$. The BMI of the non-grasp group was $21.54 \pm 3.11$. The Differences between the two 
Table 1. Comparison of shoulder stabilizer muscle activation in each group at each weight (unit : \%RVC)

\begin{tabular}{lcccccc}
\hline Muscle & & Group & $1 \mathrm{~kg}$ & $1.5 \mathrm{~kg}$ & $2 \mathrm{~kg}$ & $2.5 \mathrm{~kg}$ \\
\hline Upper Trapezius & $*$ & Grasp & $113.32 \pm 17.03$ & $138.08 \pm 27.55$ & $144.32 \pm 27.90$ & $156.72 \pm 38.13$ \\
& & Non-Grasp & $118.18 \pm 14.89$ & $128.39 \pm 30.03$ & $138.68 \pm 22.73$ & $149.56 \pm 26.18$ \\
\hline Deltoid Middle & $*$ & Grasp & $105.40 \pm 22.93$ & $119.22 \pm 30.09$ & $127.12 \pm 35.59$ & $136.84 \pm 48.37$ \\
& & Non-Grasp & $104.23 \pm 18.19$ & $110.54 \pm 36.02$ & $127.54 \pm 42.61$ & $132.51 \pm 52.65$ \\
\hline Serratus Anterior & $*$ & Grasp & $104.35 \pm 22.55$ & $128.00 \pm 24.15$ & $141.68 \pm 36.54$ & $156.42 \pm 45.98$ \\
& & Non-Grasp & $128.00 \pm 24.15$ & $108.80 \pm 35.82$ & $122.54 \pm 35.24$ & $120.84 \pm 26.29$ \\
& & & & & & \\
\hline Rhomboid & $*$ & Grasp & $109.38 \pm 9.93$ & $126.63 \pm 15.43$ & $139.19 \pm 26.41$ & $142.31 \pm 25.76$ \\
& & Non-Grasp & $105.55 \pm 15.51$ & $115.65 \pm 28.49$ & $122.04 \pm 14.72$ & $127.72 \pm 21.21$
\end{tabular}

Mean \pm SD. *: p-value are for weights, ${ }^{\dagger}: \mathrm{p}$-value are for groups.

groups were not significant. The experimental processes were sufficiently explained to the subjects, and written agreements indicating voluntary consent to participation in the experiment was received from all the subjects.

The non-grasp group was prevented from using their finger flexor muscles by fixing weight bags with diverse weights $(1,1.5,2$ and $2.5 \mathrm{~kg})$ on the backs of their hands while they were executing shoulder abduction and adduction. The grasp group was made to use their finger flexor muscles by having them grasp soft weight balls (Hygenic Corp, U.S.A.) of the same weights (1, 1.5, 2 and $2.5 \mathrm{~kg}$ ) as those of the non-grasp group while executing shoulder abduction and adduction. The activity of each muscle was measured while $0^{\circ}-166^{\circ}$ abduction and adduction of the shoulder complex were being executed ${ }^{8)}$. The measurement was repeated three times for each weight. The average values of the measured values were used. To avoid the effect of muscle fatigue, the weights were used randomly and a five-minute resting time was provided between each set of the exercises.

For electromyograms were measurements, hairs in the sensor attachment areas were removed using a shaver, keratin in the areas was removed using paper sand, and the areas were swabbed with alcohol cotton wool before attaching the sensors, in order to collect accurate data. The dominant upper trapezius, deltoid middle fiber, serratus anterior and rhomboid muscles were selected from among the shoulder stabilizer muscles. To measure the activity of each muscle, a ProComp Infiniti ${ }^{\mathrm{TM}}$ (Thought Technology Ltd., Canada) was used. Surface electrodes (Triode surface electrode, Thought Technology Ltd., Canada) consisting of three poles (Positive-Ground-Negative) were used as electrodes. The frequency range of electromyogram bandpass filtered was set to $20-500 \mathrm{~Hz}$, and the sampling frequency was set to $1,024 \mathrm{~Hz}$. The root mean square value of each muscle was measured for five seconds at the anatomical position. Relative amounts of muscle contraction to $100 \%$ of the mean of the amounts of electromyogram signals measured during three seconds, excluding the first and last one second, were calculated to show the muscle activation appearing while executing the exercises as \% reference voluntary contraction (RVC).
The measured data were analyzed with SPSS 12.0 KO (SPSS, Chicago, IL, USA) statistical software and the collected data are presented as mean and standard deviation. The significance test of the difference between the two groups and weights was tested by two-way ANOVA. The statistical significance level, $\alpha$, was chosen as 0.05 for all data.

\section{RESULTS}

The degrees of shoulder stabilizer muscle activation resulting from the use of the finger flexor muscle in relation to different weights were compared. Based on the results, both the grasp group and the non-grasp group showed tendencies of shoulder stabilizer muscle activation that increased as the weight increased. The sizes of the muscle activation increases in relation to the increases of weight were larger in the grasp group in for cases of the serratus anterior and the rhomboid. In the upper trapezius, deltoid middle fiber, serratus anterior and rhomboid, muscle activation significantly increased as the weight increased $(p<0.05)$. The serratus anterior and rhomboid showed differences between the two groups $(\mathrm{p}<0.05)$. However, it was shown that there was no interaction between the weights and the groups $(p>0.05)$ (Table 1).

\section{DISCUSSION}

Among the diverse diseases of the shoulder complex, winging scapula resulting from serratus anterior weakness is a representative injury due of muscle weakness. Studies of diverse serratus anterior muscle strengthening exercises as a way to reduce winging scapula have been conducted ${ }^{9)}$. Exercises in open kinetic chains are actively studied, reports in the literature on the forms of the hand or the use of muscular power in the distal parts during exercises in open kinetic chains are rare.

In this study, the effects of the contraction of the finger flexor muscle on the activity of muscles around the scapula were examined. Based on the results, I was seen that as the weight increased, the muscle activation of the upper trapezius, deltoid middle fiber, serratus anterior and 
rhomboid muscles increased in both groups. The serratus anterior muscle and the rhomboid muscle, that could cause swinging scapula, showed larger increases in muscle activity in relation to weight increases in the group that used finger flexor muscle contraction. These increases are thought to be the result of the preceding contraction of the shoulder complex stabilizer in order to make the finger movements. This result is consistent with the results of preceding studies, indicating that muscle activation in proximal parts is necessary for the movements of distal parts. Muscle activation around the shoulder complex in the group that used hand movements was observed. The results obtained were consistent with the results of preceding studies, indicating that weight loads imposed by grasping showed higher muscle activation ${ }^{10)}$.

The serratus anterior muscle and the rhomboid muscle showed similar degrees of contraction between cases when the grasp group, that used finger flexor muscle contraction, conducted abduction-adduction while holding $1.5 \mathrm{~kg}$. In cases where the non-grasp group, that did not use finger flexor muscle contraction, conducted abduction-adduction while holding $2.5 \mathrm{~kg}$, indicating that abduction-adduction using the finger flexor muscle induced higher activity in the shoulder stabilizer muscles with lighter weights. This result means that in the exercises of the shoulder joint and scapular stabilizer muscles, load applications activating the finger flexor muscle are more effective. Thus, shoulder stabilizer muscle exercises using the activity of the finger flexor muscle are thought to be a more effective method for strengthening the shoulder stabilizer complex.

\section{REFERENCES}

1) Williams PL: Gray's anatomy. 38th ed. Churchill Livingstone, 1995.

2) Sahrmann SA: Diagnosis and treatment of movement impairment syndrome. Mosby, 2002.

3) Bleuse $\mathrm{S}$, Cassim F, Blatt JL, et al.: Vertical torque allows recording of anticipatory postural adjustments associated with slow, arm-raising movements. Clinic Biomech, 2005, 20: 693-699.

4) Bleuse $\mathrm{S}$, Cassim F, Blatt JL, et al.: Effect of age on anticipatory postural adjustments in unilateral arm movement. Gait Posture, 2006, 24: 203-210.

5) John J, Brems MD: Rehabilitation after total shoulder arthroplasty: current concepts. Seminars in Arthroplasty, 2007, 18: 55-65.

6) Kim BG, Gong WT, Lee SY: The effect of shoulder muscle activity during visual Feedback push up-plus exercise for winging scapula. Journal of Physical Therapy Science, 2010, 22(in press).

7) Ham YW: Effects of theraband exercise on isotonic strength of flexor muscles in upper extremities. Journal of Health Science \& Medical Technology, 2000, 26: 49-56.

8) Wickham J, Pizzari T, Stansfeld K, et al.: Quantifying 'normal' shoulder muscle activity during abduction. J Electromyogr Kinesiol, 2010, 20: 212 222.

9) Voight ML, Thomson BC: The role of the scapular in the rehabilitation of shoulder injuries. Journal of Athletic Training, 2000, 35: 364-372.

10) Antony NT, Keir PJ: Effects of posture, movement and hand load on shoulder muscle activity. J Electromyogr Kinesiol, 2010, 20: 191-198. 